\title{
Communication is the Key to Success in Pragmatic Clinical Trials in Practice-based Research Networks (PBRNs)
}

\author{
Susan Bertram, RN, MSN, Deborah Graham, MSPH, Marge Kurland, RN, BSN, \\ Wilson Pace, MD, Suzanne Madison, MPH, and Barbara P. Yawn, MD, MSc
}

Effective communication is the foundation of feasibility and fidelity in practice-based pragmatic research studies. Doing a study with practices spread over several states requires long-distance communication strategies, including E-mails, faxes, telephone calls, conference calls, and texting. Compared with face-to-face communication, distance communication strategies are less familiar to most study coordinators and research teams. Developing and ensuring comfort with distance communications requires additional time and use of different talents and expertise than those required for face-to-face communication. It is necessary to make sure that messages are appropriate for the medium, clearly crafted, and presented in a manner that facilitates practices receiving and understanding the information. This discussion is based on extensive experience of 2 groups who have worked collaboratively on several large, federally funded, pragmatic trials in a practice-based research network. The goal of this article is to summarize lessons learned to facilitate the work of other research teams. ( $\mathrm{J}$ Am Board Fam Med 2013; 26:571-578.)

Keywords: Clinical Trial, Communication, Practice-based Research Networks

All large, multisite, randomized controlled trials (RCTs) require careful attention to communication among what are often geographically dispersed sites. Within a traditional RCT, which is commonly based in an academic center, communication is usually facilitated by paid full- or part-time study coordinators with no overlapping responsibilities for patient care. ${ }^{1-3}$ Most often study coordinators are people with previous experience with research and have time budgeted to facilitate communication within the site and between the site and the central project manager. ${ }^{1-3}$ However, the development of a formal communication plan is often over-

This article was externally peer reviewed.

Submitted 31 December 2012; revised 20 March 2013; accepted 27 March 2013.

From the Department of Research, Olmsted Medical Center, Rochester, MN (SB, MK, SM, BPY); and the National Research Network, American Academy of Family Physicians, Leawood, KS (DG, WP).

Funding: none.

Conflict of interest: none declared.

Corresponding author: Barbara P. Yawn, MD, MSc, Department of Research, Olmsted Medical Center, 210 Ninth Street SE, Rochester, MN 55904 (E-mail: yawnx002@umn.edu). looked. ${ }^{1}$ While previously published work on the development and implementation of communication plans in a traditional RCT may be useful, their stakeholders and messages are often quite different from those required in a pragmatic trial based in a primary care practice. Pragmatic trials assess effectiveness in a setting to optimize external validity or generalizability. A pragmatic trial enrolls the usual primary care patient from a community practice rather than enrolling only those patients meeting extensive inclusion and exclusion criteria into a tightly controlled research process. ${ }^{4,5}$

Communication required for implementation and completion of a pragmatic RCT in a practicebased research network (PBRN) is a complex process requiring several unique considerations related to the PBRN practice sites. PBRN sites often have limited experience with research and rarely include staff to facilitate research within the site. Regional PBRNs have developed positions for people to facilitate research in geographically linked practices. Practice enhancement assistants ${ }^{6}$ or practice enhancement and research coordinators ${ }^{7,8}$ are feasible 
for regional or local PBRNs but are difficult to use in widely dispersed national trials. The presence of practice enhancement assistants or practice enhancement and research coordinators may also hinder the pragmatic nature of the research, adding staff to the practice who are not usually present and will not be available after the study is complete. While US-based groups such as Clinical \& Translational Science Award sites and the National Institutes of Health are encouraging pragmatic trials, ${ }^{4,9,10}$ little has been published to describe successful communication strategies for those who design and implement large, national, practicebased pragmatic trials. ${ }^{8}$

This article describes the experience of 2 groups, staff at the Olmsted Medical Center (OMC) Department of Research and the American Academy of Family Physician's National Research Network (AAFP NRN), in implementing and supporting the completion of several pragmatic studies. We believe the information presented here will allow others to avoid pitfalls we encountered, to adapt solutions we developed, and to facilitate dialogue within the T3 translational (moving evidence from the bedside to community practice $)^{11}$ and pragmatic trials research communities.

\section{Study Overview}

The 2 studies from which this information is taken were trials that introduced practice system changes related to postpartum depression and asthma care to a group of "intervention practices" while also collecting data from "usual care practices." For each site enrolled in each of the studies, a lead physician and a lead nursing person were identified and participated in a centralized study training program. Those 2 individuals then trained the rest of the members at their practice. The studies continued for a period of 18 to 48 months, and in each study the usual care sites crossed over to intervention practices after a period of 12 to 24 months. PBRN practice sites spanned the United States and included a spectrum of practices, from urban private practices and university-affiliated urban family medicine residencies to small rural practices and several federally qualified health centers.

\section{The Central Research Team}

Investigators and research staff from the Olmsted Medical Center (OMC) Department of Research
(Rochester, MN), from the American Academy of Family Physicians National Research Network (AAFP NRN) in Leawood, KS, with the Director based in Denver Colorado and from Dartmouth College in Hanover, New Hampshire, collectively, were the central study team. Members of this group were based in four states and three time zones, requiring a communication approach, job description, and coordination plan for the central team.

The central team developed a series of weekly conference calls; one 60-minute call for all central team members and a second 30-minute call for the lead study coordinators from OMC and the AAFP NRN. The weekly calls with all central team members provided everyone with study updates and highlighted issues requiring the development of a team strategy and solutions to issues such as inadequate patient enrollment and the failure of specific clinics to systematize the intervention and tracking of institutional review board activities. The coordinator calls assured that decisions were understood among the central team members, supporting consistent messaging to the practice sites and consistent implementation of strategies. By 12 to 15 months into the first study, the central research team developed into a cohesive group, one that encouraged and valued input from all members. When working across multiple time zones, study staff must be flexible and recognize that meetings may start before or end after typical work hours. Scheduling a time to meet can be challenging. Instead of looking for open times for all team members, look for times that can be freed up with advance planning as well as currently available times. Recognize the team is planning for 3 to 5 years of meetings and plan accordingly. If the time will work in 6 or 8 weeks for a member of the team, then consider it a viable option. Over the course of a major study people will miss meetings, but the meetings should generally proceed anyway. Our teams have not had problems with poor attendance at the meetings (various members of our groups have been meeting at the same time and day of the week for more than 7 years across various studies), but if attendance by a relevant individual is a problem then a conversation with the individual and the primary investigator (PI) of the study or the local site would be in order.

As Internet technology advanced, new methods of interaction among the central team were used, including a secure shared website for all team mem- 


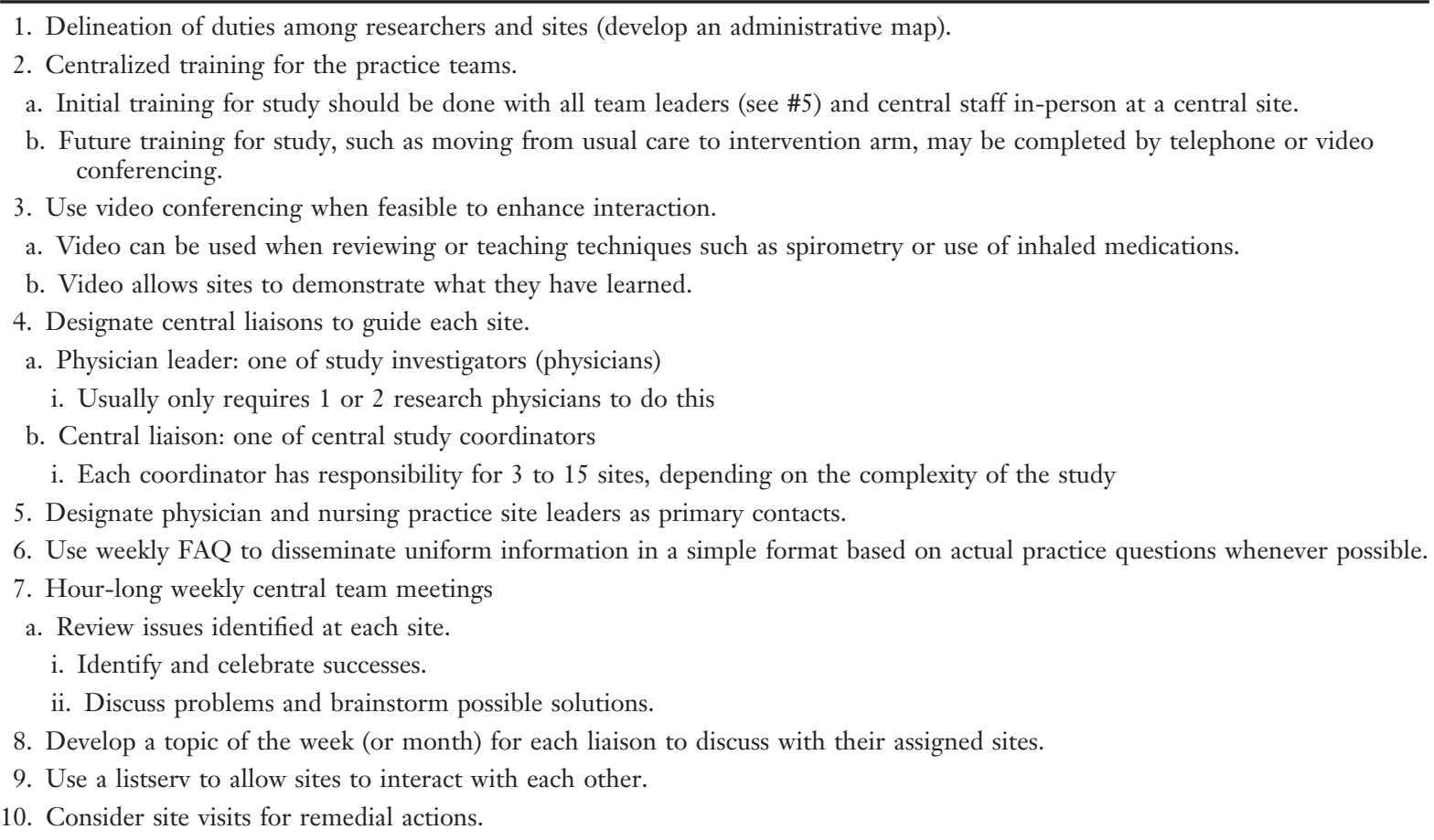

bers that was housed at the University of Colorado, Denver, an AAFP NRN partner. This technology was especially useful when updating practice tools and editing manuscripts. Yearly or bi-yearly inperson meetings of the entire central research team further facilitated the conduct of the study, specifically data analysis and interpretation and manuscript development. Such meetings were held in conjunction with other national meetings that members of the research team were likely to attend or at the OMC, where the largest portion of the team worked. Meetings of the research team should be included in the study budget. Our groups have never had these types of meetings questioned by review committees.

\section{Study Sites}

Forty-nine clinics participated in the 2 studies whose communication methods are described here, and Table 1 describes many of the tools used to facilitate communication. Communication with study sites began at the central face-to-face training sessions, when the lead physician and site coordinator were introduced to the entire central study team. The central team included 2 or 3 senior investigators, a project manager (who also functioned as one of the site liaisons), and 3 other part-time study liaisons. To main- tain a personal connection between the central team and the clinics, each site was assigned a PI and a central team liaison to guide the clinic's progress during the study. These 2 people (one of the overall study PIs and a site liaison) acted as the clinic's primary contacts during the years they were involved in the study.

Pictures of the leads at each site were taken during training, as were photographs of the central team members. Each site received pictures of their liaison, and the pictures of the site dyads were attached to a US map kept at the central team office to help connect names and faces to their locations. The face-to-face meeting facilitated establishing personal relationships between the central study personnel and each clinic's team as well as fostering a sense of a research community among all sites. Sites offered suggestions for adapting and improving the research protocol during these meetings, which helped with local ownership of the project. Both the OMC research group and the AAFP NRN have used a centralized training approach for all significant studies (those done together as well as those led by each separately) for more than a decade. The interaction between practices and the adjustments and improvements to the study protocol are valuable. The need for the central research 
team to carefully plan a prolonged educational activity sharpens the educational focus and improves the materials for educational activities of the full office staff.

\section{Interactions between the Central Research Team and Practice Sites}

Central team liaisons communicated weekly with each site coordinator. The calls were designed to be brief, goal-oriented, and with content standardized across sites supplemented with individually tailored messages. Most calls focused on implementing the study and sustaining practice system change. The weekly talking points were determined during the calls of the central study team, which included discussions about the need for and content of messages tailored to each site's progress and perceived problems. Standardization of messages and specific plans for message delivery ensured that all sites heard the same information each week. A recurrent theme of the central team's desire to provide support and education regarding study procedures was mentioned during each call in a manner that allowed the liaison to assess protocol fidelity and discuss local challenges and successes. For example, the liaisons might ask which of the site physicians'/ clinicians' patients were enrolled in the study to assess how widespread study participation was among the staff. Other questions might address the use of one of the practice change tools, asking how often the tool was being used by each of the staff members. Solutions to identified challenges were facilitated during the calls, and sites often developed solutions with active listening from central liaisons. Successes were shared with all clinics through the standardized messages.

The central team liaisons maintained and supported the approach that patient care comes first by being flexible and cooperative in scheduling calls and rescheduling missed calls. The central team liaisons adapted their schedules in response to the needs of the site coordinators. The ability to set flexible call times and always work with clinics to increase the efficiency of study procedures demonstrated our recognition that research was not the primary focus of the clinics and that patient care took priority. Communicating this attitude through actions as well as words (eg, scheduling calls with the site team and always checking to make sure the identified call time is still a "good time to talk" when beginning the call) is a critical step in gaining the collaboration of busy primary care health professionals.

E-mail or text messaging was a quick way to communicate needed information and/or to reschedule missed calls. Although calls and emails were directed primarily to the site coordinators, we encouraged the site coordinators to discuss study issues or concerns with their clinic's lead physician and the site study team to engage other clinic personnel in the discussion of study problems and progress.

The study called the sites' lead physicians on a monthly basis to help maintain interest in the study and provide support to the lead physician, who may have been participating in their first PBRN study. These contacts emphasized the important role of the lead physician in maintaining the clinic's investment and enthusiasm for that site's success in the study. The study PIs also called site staff when the site liaisons were having trouble reaching site coordinators. Such calls focused on the ways that might be used to free some of the site coordinator's time for study activities and assess any major practice issues, such as introduction of a new electronic medical record, which occurred in several sites and moved the study into the background.

When a person other than one of the 2 team leaders performed a special study activity (eg, copying medical records or making calls to engage patients), the central liaison for that site arranged for monthly calls with the individual performing the special study task. The purpose of these calls was to reinforce the role the individuals played in the study and its importance for the success of the study. It also provided an opportunity to review the extent to which the intervention clinics were adhering to the intervention, discuss what problems they were having, and encourage the individuals to talk to the site leadership team. With our assistance, several sites created their own tools, such as follow-up charts or registries to help staff remember the steps of the intervention. With permission from the site, the tools were shared with all other clinics. Use of these site-developed tools, as well as the practice change study tools, was assessed by medical record review of visits after the intervention.

Relationships with the lead physician and site coordinator were built over time via these phone calls, E-mails, and frequently asked questions 
(FAQs). We learned not only how the clinics were progressing in the study but also of personal milestones such as engagements, weddings, major events related to children, births, and illnesses. We celebrated joyous events and remembered individuals in times of sadness with letters and cards. We heard from them that our cards of sympathy and best wishes were displayed in their offices. We sent electronic cards to celebrate events such as enrollment achievements and the coordinators making it through a particularly difficult week at the site.

All sites also received a weekly communication called the FAQ, which was transmitted by fax and E-mail listserv to each study site. We asked the site to post the FAQ in a central location to facilitate communication of study specifics to all clinic personnel. The FAQs reinforced messages communicated by phone calls and provided the sites with a written record of important study points.

\section{Tools to Enbance Communication}

In a PBRN research protocol with multiple study sites and personnel with widely varying levels of research experience and expertise, each step of study implementation and monitoring should be guided by a communication plan that includes both content and process tailored to the practice site. Focused attention on communication can facilitate building strong relationships between the central team members and staff of the study clinics.

The content and the method of communication require dedicated planning. The operational definition of study-related terms needs to be developed and shared. For example, for the postpartum depression study, a woman should be invited into the study at "any nonemergent visit a woman has in the period of 4 weeks to 12 weeks after she has delivered a potentially viable baby." It provides the time frame, comments on the emergent nature of the visit, and excludes patients who have had miscarriages or abortions. The basic message is consistent across all sites and readily lends itself to more in-depth explanation that also will be consistent.

To maximize access and allow for emergent communication, a toll-free phone line dedicated to the study was established and provided clinics and enrollees a way to call us without charge. Clinic practices were encouraged to contact the central team at any time on the toll-free line, which was answered 24 hours a day, 7 days a week; OMC study staff rotated to provide after-hours coverage.
A clinic occasionally asked for after-hours advice or support and patients called with a variety of questions. Although the weekend and evening calls usually were answered by a physician, no medical advice was given except in one instance when 911 was called for a patient in extreme distress.

E-mail listservs were established (intervention and control listservs) to allow us to send E-mails as a mass communication to all lead physicians and study coordinators and enabled the clinics to communicate with each other. A few of the sites communicated with each other outside of the shared listserv. This interaction was limited but might be valuable to strengthen in future studies.

\section{Communication Challenges}

Some messages and study procedures required repeated or alternative explanations. Local sites occasionally ignored our routine calls and E-mails. Communication problems were discussed during our weekly central team meeting for support as well as advice on how best to proceed. Tips were shared between central liaisons on the best day or time to contact a study site. The central team members reminded the site liaisons that a lack of response was not a personal affront and might indicate anything from a natural disaster (flood or tornado) to sudden illness to embarrassment regarding a misunderstood FAQ. Sometimes communication with the lead study physician by one of the physician investigators was used to reestablish lapsed communication with a site. Over the course of the studies, only 3 of the total 49 sites failed to respond within 2 weeks to one form or another of communication with the central team.

Site visits were made by the PI or co-PI when sites continually had difficulty with study implementation and regularly missed calls and interactions. One of the physician investigators went to a practice site and not only worked with the lead study physician and site coordinator but also met with as many staff members as possible. Providing lunch facilitated staff attendance. It was useful for clinic personnel to hear the investigator offer suggestions of how to best organize the study within the practice, and it was likewise helpful for the central site to understand more specifically how the clinic functioned. After one site visit, the lead physician was inspired to put the informational flyer about the study procedures in the staff bathroom so everyone would 
Table 2. Lessons Learned from Pragmatic Trials in Practice-based Research Network (PBRN) Clinics

\begin{tabular}{|c|c|}
\hline Actions & Implementation \\
\hline Create a communication plan & $\begin{array}{l}\text { Create a plan that includes a plan for the flow of communication between the central team } \\
\text { members and clinic sites and for the dissemination of information to the central team } \\
\text { and all clinic sites. }\end{array}$ \\
\hline $\begin{array}{l}\text { Know what message you want to } \\
\text { communicate }\end{array}$ & $\begin{array}{l}\text { Be brief. Stick to the message you wish to convey. Repeat the message. Do not expect all } \\
\text { messages to be heard and understood the first time they are communicated. }\end{array}$ \\
\hline Be respectful of time & $\begin{array}{l}\text { Physicians, nurses, and clinics are primarily engaged in the care of patients. Set meeting } \\
\text { times in advance. Be on time. Be understanding if a meeting must be rescheduled. }\end{array}$ \\
\hline $\begin{array}{l}\text { Identify each site's preferred } \\
\text { communication mode and use it }\end{array}$ & $\begin{array}{l}\text { Ask how the physician or nurse prefers you to communicate with them. Not everyone } \\
\text { views E-mails throughout the day. Use listservs, FAQs, text or phone messaging, faxes. } \\
\text { Provide all with your phone number, E-mail address, and fax number with each } \\
\text { electronic communication. }\end{array}$ \\
\hline Pictures are worth 1000 words! & $\begin{array}{l}\text { Pictures in E-mails help convey your message. Use maps and a contact list with photos to } \\
\text { help you remember to whom you are speaking. Use a clock to mark the time zones } \\
\text { where clinics are located. Send photos. Ask for photos from the clinic when you send } \\
\text { treats or buy them lunch. }\end{array}$ \\
\hline Make phone calls free & $\begin{array}{l}\text { Establish toll-free phone line for patients enrolled in the study and clinic staff. Be available } \\
\text { to sites } 24 \text { hours a day if necessary for site calls regarding concerns, especially } \\
\text { medication issues. Maintain a study E-mail for patients. }\end{array}$ \\
\hline Be flexible but consistent & Be persistent but patient. Convey your message in a palatable way. \\
\hline Celebrate successes, big or small & $\begin{array}{l}\text { Send cards, eCards, E-mails to site coordinators and lead physicians. Congratulate all } \\
\text { successes! Notify clinics when they have reached goals or done something out of the } \\
\text { ordinary. Share in the personal successes of individual physicians, nurses, and clinics. } \\
\text { Provide certificates when goals are reached. Send small rewards throughout the year to } \\
\text { encourage continued good will. }\end{array}$ \\
\hline Expect change-it is inevitable & $\begin{array}{l}\text { Be prepared for personnel changes within the central team and at the clinic sites. Plan for } \\
\text { training of personnel who are new to the study. }\end{array}$ \\
\hline Set realistic expectations & $\begin{array}{l}\text { Do all that you can to help everyone to succeed, but be prepared that not all will be } \\
\text { successful. }\end{array}$ \\
\hline
\end{tabular}

FAQ, frequently asked questions.

be able to regularly view the study information; enrollment increased afterward. Overall, for the 2 studies, 3 site visits were made to try to improve study activity in an enrolled practice. Not all site visits solved the problems: 6 practices of a total of 55 enrolled and 49 completing practices (11\%) prematurely ended their involvement in the study.

\section{Discussion of Lessons learned}

Practice-based research or pragmatic trials require special attention to communication. Over the course of 2 large nationally distributed studies we have learned several important planning lessons; these are shared in Table 2.

Published outlines for developing a communications plan can be a useful beginning. It is important to identify with whom you must communicate (stakeholders) and what they need to hear during and learn from those communications, determine how the communication needs are to be met, and revise and share the plan as necessary. ${ }^{3}$ While an outline is useful, the process of filling in the outline is seldom discussed in the literature. The stake- holders may seem obvious, but in a pragmatic study any practice member-from the reception staff to the phlebotomist-may need to become a recipient of communication regarding the general purpose and requirements of the study. The methods of delivering communication are seldom as simple as deciding whether electronic or hard copy formats are required. ${ }^{1}$

Our lessons learned are accompanied by our overview of tools to address communication issues. Similar to others who have published communication strategies, we agree that delineating roles and responsibilities is required. ${ }^{1,3}$ In a practice-based study, the title of the person with a specific responsibility may vary across sites. For example, what may be a nursing responsibility at one site may be the responsibility of a receptionist, pharmacist, or clinic manager at another site. Therefore, communication must be relevant to the responsibility, not to the title.

Centralized face-to-face training is highly recommended for all trials. The model of centralized training should be considered for regional network 
studies even if visitation to each site by study staff is possible. In pragmatic or PBRN trials, staff at the sites are unlikely to have met each other, and if it is a national network, site physicians may not have met the study leaders. Centralized training facilitates rapport and allows training to occur in a sequestered environment, allowing the practice teams to focus on the study without the multitude of disruptions that can occur when training occurs in a clinical setting and lasts longer than 60 minutes.

While the communication plan and strategies must be flexible to fit the needs of the practices, some standardized tools can facilitate continuity and consistency. We found the weekly FAQ to be one of our most effective tools to encourage responses from the practice sites and share the same information with everyone. Listservs can be used to post the FAQs and support site interactions. Receiving feedback from the sites facilitates the process of tailoring and improving our message crafting.

Interactions among the central sites may be needed on an ad hoc basis to address any urgent issues. However, having planned weekly conference calls that include the central liaisons, study managers, and the PIs assures that everyone is kept up to date. An agenda and repetitive updates on important topics (eg, weekly enrollment, questionnaire return rates, and institutional review board yearly submission status) assures that every member of the central study team has the basic information they need to interact accurately with sites and each other. While we believe the information presented is practical and applicable to most pragmatic studies based in PBRNs, the lessons we present are seen through the eyes of 2 teams that have worked together for more than 10 years. It is likely that we have failed to include many of our early lessons since we did not begin recording them until we were about half way through the second study. In addition, our practices are family medicine practices that may have fewer support staff members and fewer nursing staff at the licensed practice nurse or registered nurse levels than subspecialty practices. For example, many of the nursing staff members were nursing assistants who had never participated in a clinical research study. Studies with primarily academic medical center sites may be less likely to be involved in pragmatic trials and likely to have different communication issues. Therefore, our work might not be generalizable to all PBRN studies. Not all the solutions proposed in Tables 1 and 2 worked for all sites. However, it is important to have a variety of approaches, and that is what we have provided here.

\section{Feasibility}

The techniques we describe were used during the conduct of large federally funded studies with 20 to 30 practices per study, annual budgets of $\$ 400,000$ to $\$ 500,000$, and widely dispersed practices. Each study provided funding for 3 full-time equivalents (FTEs) of study liaisons/coordinators, 2 FTEs from OMC and 2 part-time FTEs from the AAFP NRN. Both trials were led by a PI who devoted significant time (0.3 FTE) to each of the studies, allowing the regular interaction of all study team members. Funding for central training averaged about $\$ 1,000$ per attendee ( 2 attendees per practice) and covered airfare, lodging, and meals. The funding for this activity was divided over 2 years of the grants' funding. Working at a national level, the financial cost of visiting 30 practices, as well as the toll on study staff, is as great as or greater than that of centralized training activities. Dedicated phone lines were added to the existing phone service of the OMC and cost approximately $\$ 1,200$ per year. While 24-hour phone coverage might seem difficult, the study PI did provide this service with support from senior study coordinators. Enrolled practices reported that this support was reassuring even if it was seldom used. Practicing physicians and their staff seemed to be careful to use this service only when an immediate answer or assistance was required. When access to the central team is required 24 hours a day, it is important to consider the nature of the information that is needed in real time and the personnel who can provide the information. For instance, a study involving atypical treatments with blinded medications may require immediate contact with a physician. For many other studies a member of an informed research team can provide the needed support or information as well as a physician.

\section{Conclusion}

PBRN research is a collaborative effort, and the central research site(s) must use regular communication to assist the practices in implementing the study based on the balance of protocol requirements and local needs and resources. Communica- 
tion includes the process of delivering as well as crafting the message. Tailoring messages and developing multiple communication approaches increases the likelihood of successful implementation of the protocol and completion of the study. Messages from the central research site should be consistent, short, clear, and repeated regularly and in a variety of ways. Remembering that research communications are heard by PBRN practices within the context of a clinic tasked primarily with providing patient care can facilitate the flexibility and patience of the research team. Successful communications are not just received and understood by the "average" practice but by all sites in a way that leads to study fidelity.

\section{References}

1. Richardson B, Newman R. Clinical trial management-an integrated process. 2006. Available from: http://edp316745.wikispaces.com/file/view/Clinical+ Trial+Planning-1.pdf. Accessed July 17, 2013.

2. Halloran L. Clinical study project plans. J Clin Res Best Pract 2010;6:1-6.

3. Mahinda T. Back to basics developing an effective communication plan for clinical trial management. Monitor 2006:19-23.

4. Patsopoulos N. A pragmatic view on pragmatic trials. Dialogues Clin Neurosci 2011;12:217-24.
5. Godwin M, Ruhlan L, Casson I, et al. Pragmatic controlled clinical trials in primary care: the struggle between external and internal validity. BMC Med Res Methodol 2003;3:28.

6. Aspy C, Enright M, Halstead L, Mold JW; Oklahoma Physicians Resource/Research Network. Improving mammography screening using best practices and practice enhancement assistants: an Oklahoma Physicians Resource/Research Network (OKPRN) study. J Am Board Fam Med 2008;21:326-33.

7. Fagnan L, Dorr D, Davis M, et al. Turning on the care coordination switch in rural primary care: voices from the practices-clinician champions, clinician partners, administrators, and nurse care managers. J Ambul Care Manage 2011;34:304-18.

8. Pace W, Fagnan L, West D. The Agency for Health Care Research and Quality(AHRQ) Practice-Based Research Network (PBRN) relationship: delivering on a opportunity, challenges, and future directions. J Am board Fam Med 2011;24:489-92.

9. Kroenke K, Kapoor W, Helfand M, Meltzer D, McDonald M, Selker H. Training and career development for comparative effectiveness research workforce development. Clin Transl Sci 2010;3:258-62.

10. Zwarenstein M, Treweek S, Gagnier J, et al; CONSORT Group; Pragmatic Trials in Healthcare (Practihc) Group. Improving the reporting of pragmatic trials: an extension of the CONSORT statement. BMJ. 2008;337:a2390.

11. Woolf SH. The meaning of translational research and why it matters. JAMA 2008;299:211-3. 